\title{
Dynamic Range Improvement of IM-DD Optical Link using Dual-Wavelength Dual-Parallel Modulation
}

\author{
Li Xianghua, Yang Chun*, Zhou Zhenghua, and Chong Yuhua \\ School of Electronic Science and Engineering, Southeast University, Nanjing 210096, Jiangsu Province, China
}

(Received April 8, 2014 : revised June 9, 2014 : accepted June 9, 2014)

\begin{abstract}
In this paper, we present an incoherent dual-parallel modulation linearized optical link employing two different optical wavelengths. Comparing with previous dual-parallel modulation, this linearization method prevents the recreation of distortion products and can be readily implemented. Furthermore, a dual-wavelength dual-parallel modulation linearized optical link constructed with the commercially available devices is experimentally demonstrated with a spurious-free dynamic range of $122.5 \mathrm{~dB} . \mathrm{Hz}^{4 / 5}$. More than $25 \mathrm{~dB}$ suppression of the intermodulation distortion, $15.6 \mathrm{~dB}$ improvement of spurious-free dynamic range and $1.5 \mathrm{~dB}$ improvement of compression dynamic range are achieved after linearization.
\end{abstract}
Keywords: Analog optical link, Linearization method, Intermodulation distortion, Spurious-free dynamic range
OCIS codes : (060.4510) Optical communications; (230.4110) Modulators; (060.2360) Fiber optics links and subsystems

\section{INTRODUCTION}

The use of analog optical links to transmit or distribute the radio frequency (RF) has attracted a great deal of attention over the past decades, due to its advantages of large bandwidth, low loss, and immunity to electromagnetic interference. Analog optical links have a host of commercial and military applications, such as cable television, radio over fiber, remote antenna, satellite communications and phase-array radars [1]. The most common scheme of analog optical links is intensity modulation and direct detection (IMDD) employing an external Mach-Zehnder modulator (MZM), which is free of the resonance distortion and provides a larger bandwidth than the direct modulation of a laser.

A key characteristic of the analog optical links is the spurious-free dynamic range (SFDR) that is defined as the range of input powers in which the signal is above the noise floor and intermodulation distortion is below the noise floor [1]. Generally, the third-order intermodulation distortion (IMD3) is the main restricting factor of the SFDR, which is inherently introduced by the nonlinear transfer function of an MZM. Therefore, numerous methods have been proposed to suppress the IMD3 of the IMDD links, including predistortion linearization [2-5], polarization mixing [6-7], dual-wavelength input [8-9], dual-serial modulation [10-11] and dual-parallel modulation (DPM) [12-14]. The predistortion linearization reduces the distortion in the electronic domain, and its electric circuit is usually complicated. The polarization mixing and the dual-wavelength input lack flexibility in attaining the optimum performance, as is attributed to the use of a single MZM. The dual-serial modulation suffers the even order distortion and is limited to sub-octave applications. However, the DPM offers greater flexibility in attaining the optimum performance by using two spatially distinct modulators and can be used in multi-octave applications when the two MZMs operate at the quadrature point. The DPM has been implemented using the coherent approach [12] and in the incoherent way with a single optical source [14]. In the coherent DPM, the optical power is asymmetrically divided by a polarization-maintaining optical coupler, then fed into two MZMs respectively, and finally recombined by an optical coupler. An optical phase shifter is required to maintain the quadrature between the outputs of the two MZMs in order to prevent the recreation of distortion products [13]. Considering the high sensitivity of the optical phase on the

*Corresponding author: yangchun_seu@163.com 
disturbance of the environment, a feedback loop controller is needed to actively adjust the phase shifter. The incoherent DPM with a single optical source employs a polarization beam combiner to recombine the outputs of the two MZMs, which prevents the use of the additional phase shifter and the loop controller.

In this study, we present an incoherent DPM linearization method employing dual wavelengths. By the use of two optical sources operating at the two different wavelengths, the linearization method presented here does not require an additional optical phase shifter to maintain the quadrature between the outputs of the two MZMs [12] and avoids the recreation of intermodulation distortions due to the polarization coupling in the long-haul single mode fiber (SMF) [14]. Therefore, the dual-wavelength DPM (DWDPM) is a good alternative for the high-linearity optical links. However, this DWDPM requires the two optical sources to maintain at a constant power ratio. So the SFDR impairment caused by the optical power drifting is numerically simulated.

The rest of this paper is organized as follows: In Section 2 , the intermodulation distortions and the gain compression of a single MZM link are analyzed using Taylor's series. The operating theory of the DWDPM is also presented. In Section 3, the DWDPM is experimentally demonstrated with the commercially available components. The SFDR and the compression dynamic range (CDR) of the DWDPM linearized link and the single MZM link are measured, respectively. The conclusion is drawn in Section 4.

\section{PRINCIPLE OF OPERATION}

\subsection{The Distortions of a Single MZM Link}

Usually, a conventional single MZM link is composed of a laser, an MZM, a PD and an optical fiber. A continuouswave laser beam is first modulated by an RF signal in the MZM. After transmitting through a length of optical fiber, the laser beam is fed into the PD that recovers the RF signal from the optical carrier. Generally, the MZM is biased at the positive quadrature (+QUAD) or the negative quadrature (-QUAD) to maximize the gain of the optical link and avoid the even-order distortions. In these cases, the output photocurrent of the single MZM link can be given as below:

$$
i_{\text {out }}=I_{d c}\left[1 \pm \sin \left(\frac{\pi V_{R F}(t)}{V_{\pi}}\right)\right]
$$

where $I_{d c}$ is the DC photocurrent, $V_{\pi}$ is the half-wave voltage of the MZM, $V_{R F}(t)$ is the driving RF signal. We expand the Eq. (1) into the Taylor's series and take to the quintic term as below:

$$
i_{\text {out }}=a_{0}+a_{1} V_{R F}(t)+a_{2} V_{R F}{ }^{2}(t)+a_{3} V_{R F}{ }^{3}(t)+a_{4} V_{R F}{ }^{4}(t)+a_{5} V_{R F}^{5}(t)
$$

where $a_{i}(i=1,2,3 \ldots)$ are the coefficients of Taylor's series, normally $\left|a_{1}\right|>>\left|a_{3}\right|>>\left|a_{5}\right|$. The SFDR of the single MZM link can be derived using the conventional two-tone method. By combining the two-tone RF signal with the equal amplitude in $\operatorname{VRF}(t)$, the fundament signal and the distortions are derived based on Eq. (1) and shown in Table 1:

TABLE 1. The amplitudes of the signal and the distortions output from a single MZM link

\begin{tabular}{c|c|c|c}
\hline \hline Frequency & $\begin{array}{c}\text { Signal \&. } \\
\text { Distortions }\end{array}$ & Freq Component & Amplitude \\
\hline \multirow{2}{*}{$\omega_{1}, \omega_{2}$} & $\begin{array}{c}\text { Fundamental } \\
\text { Signal }\end{array}$ & $\omega_{1}, \omega_{2}$ & $a_{1} V_{0}$ \\
\cline { 2 - 4 } & Residual HMD3 & $2 \omega_{1}-\omega_{1}, 2 \omega_{2}-\omega_{2}$ & $9 a_{3} V_{0}^{3} / 4$ \\
\cline { 2 - 4 } & Residual HMD5 & $3 \omega_{1}-2 \omega_{1}, 3 \omega_{2}-2 \omega_{2}$ & $25 a_{5} V_{0}^{5} / 4$ \\
\hline \multirow{2}{*}{$\begin{array}{c}2 \omega_{1}-\omega_{2}, \\
2 \omega_{2}-\omega_{1}\end{array}$} & IMD3 & $2 \omega_{1}-\omega_{2}, 2 \omega_{2}-\omega_{1}$ & $3 a_{3} V_{0}^{3} / 4$ \\
\hline $\begin{array}{c}3 \omega_{1}-2 \omega_{2}, \\
3 \omega_{2}-2 \omega_{1}\end{array}$ & Residual IMD5 & $\begin{array}{c}3 \omega_{1}-\omega_{1}-\omega_{2}, \\
3 \omega_{2}-\omega_{1}-\omega_{2}\end{array}$ & $25 a_{5} V_{0}^{5} / 8$ \\
\hline
\end{tabular}

where $\omega_{i}(i=1,2)$ are the angular frequencies and $V_{0}$ is the amplitude of the two-tone RF signals. From the above table, it can be seen that the RF outputs at the fundamental frequencies $\left(\omega_{1}, \omega_{2}\right)$ are not only dictated by the fundamental signal, but also depend on the residual third-order harmonic distortions (HMD3) $\left(2 \omega_{1}-\omega_{1}, 2 \omega_{2}-\omega_{2}\right)$ and the residual HMD5s $\left(3 \omega_{1}-2 \omega_{1}, 3 \omega_{2}-2 \omega_{2}\right)$. When the RF signal input is large enough, the amplitude of the RF outputs at the frequencies $\left(\omega_{1}, \omega_{2}\right)$ are no longer linearly proportional to that of the RF input and the gain of the link becomes compressed, because $a_{1}$ and $a_{3}$ are opposite in sign and the amplitude of the residual HMD3s $\left(2 \omega_{1}-\omega_{1}, 2 \omega_{2}-\omega_{2}\right)$ increase with the $\mathrm{RF}$ input more quickly than that of the fundamental signals, moreover, the residual HMD5s are much smaller and can be neglected. The RF outputs at the intermodulation frequencies of $\left(2 \omega_{1}-\omega_{2}\right)$ and $\left(2 \omega_{2}-\omega_{1}\right)$ are dictated by the IMD3s and the residual IMD5s. The IMD3 is the contribution of the cubical term. The residual IMD5 is the contribution of the quintic term and it is much smaller than the IMD3. Therefore, every linearization method essentially diminishes $a_{3}$ so as to increase the SFDR and the CDR.

\subsection{The Dual-wavelength Dual-parallel Modulation}

The schematic diagram of the DWDPM linearized link is shown in Fig. 1. The two continuous-wave laser beams are fed into the two MZMs, and modulated by the RF signals, respectively. Then, the two modulated laser beams are combined using a wavelength multiplexer (WM), and finally injected into a PD. The primary path with a large optical power is modulated by a small RF signal, while the secondary path with a small optical power is modulated 
by a large RF power and $180^{\circ}$ out of the phase relative to the primary path. Therefore, when a two-tone RF signal is input in the DWDPM link, the carrier-to-intermodulation ratio (CIR) of the primary path is larger than that of the secondary. With the proper control of the input optical power of the second path, the IMD3 produced in the secondary path can be equal in magnitude to that produced in the primary path, while the fundamental signal of primary path is larger than that of the secondary path. Moreover, the IMD3s of both paths are opposite in phase as well as the fundamental signals of both paths. So the IMD3 of the link can be cancelled with a penalty of a little reduction of the fundamental signal. In this case, the residual IMD5 becomes the dominating intermodulation distortion and limits the SFDR of the optical link. Particularly, the two lasers operate at two different wavelengths to prevent the beatings of two MZM's outputs lying in the bandwidth of the modulated signal.

The major concern of the DWDPM linearized link is the SFDR impairment caused by the optical power fluctuation of the two lasers. In order to analyze this impairment, we

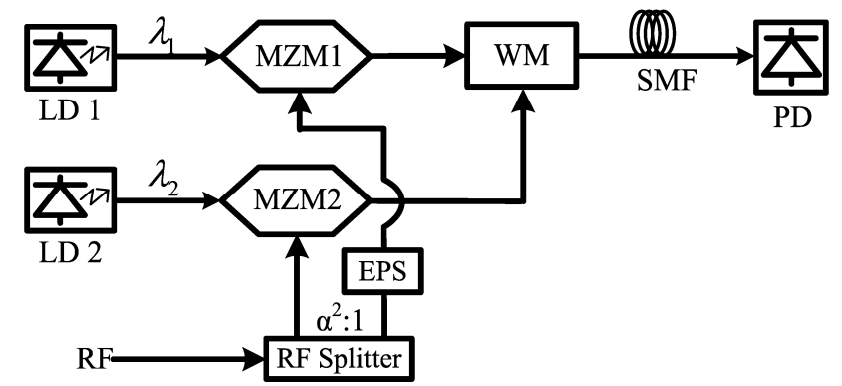

FIG. 1. Schematic diagram of the DWDPM linearized link. EPS: the electric phase shifter. WM: the wavelength multiplexer.

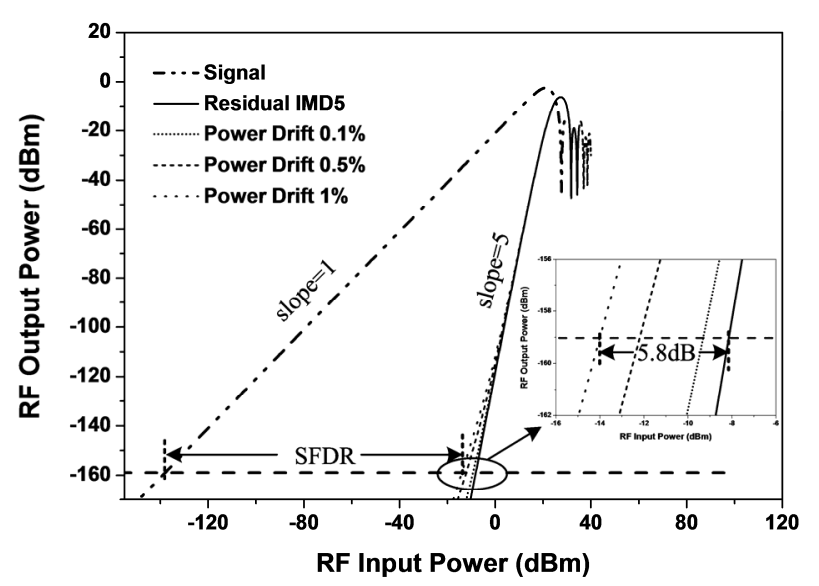

FIG. 2. SFDR of the DWDPM linearized link under shot noise limitation is exacerbated by $1.2 \mathrm{~dB}, 4 \mathrm{~dB}$ and $5.8 \mathrm{~dB}$ respectively, when the optical power of the secondary laser drifts by $0.1 \%, 0.5 \%$ and $1 \%$ while that of the primary laser is held constant. numerically simulate the SFDR of the DWDPM linearized link under the shot noise limitation with the optical power of the secondary laser drifting and that of the primary laser held constant, as shown in Fig. 2. It is indicated that the SFDR is exacerbated by $1.2 \mathrm{~dB}, 4 \mathrm{~dB}$ and $5.8 \mathrm{~dB}$ respectively, when the optical power of the secondary laser drifts by $0.1 \%, 0.5 \%$ and $1 \%$. The output-power stability of the commercial laser diode controller working in the constant power mode can attain $0.02 \%$, so a high SFDR can be obtained using the DWDPM method.

\section{EXPERIMENTAL SETUP AND RESULTS}

We experimentally compare a DWDPM linearized link with an unlinearized single MZM link. The single MZM link is constructed with an EM4 DFB laser, a Discovery DSC40S PD and a Covega MZM biased at +QUAD. The intermodulation $\left(2 \omega_{1}-\omega_{2}, 2 \omega_{2}-\omega_{1}\right)$ of the single MZM link is measured with a $10 \mathrm{GHz}$ two-tone RF signal spaced 10 $\mathrm{kHz}$, as shown in Fig. 3(a). When the output power of the fundamental signal equals $-20 \mathrm{dBm}$, the CIR of this link is

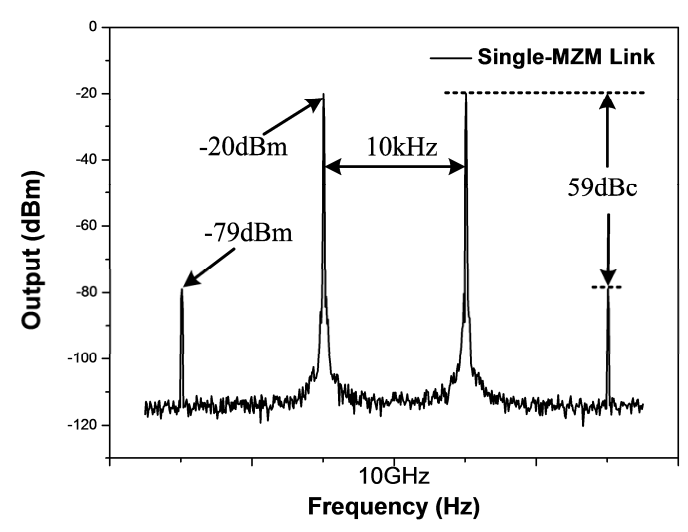

(a)

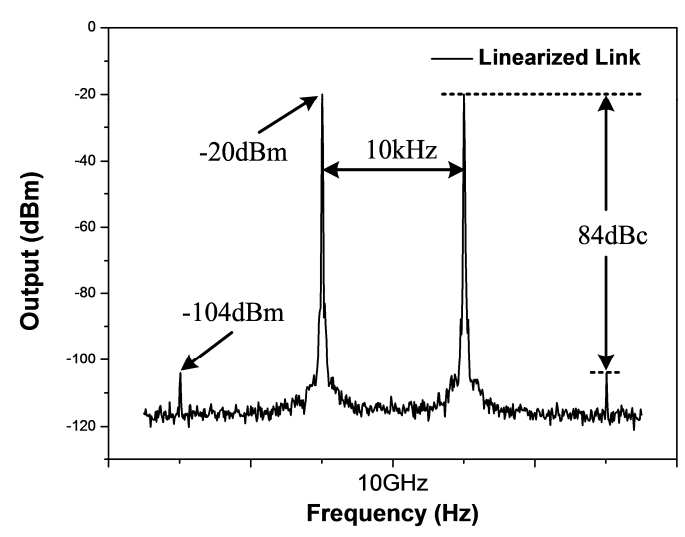

(b)

FIG. 3. The intermodulation $\left(2 \omega_{1}-\omega_{2}, 2 \omega_{2}-\omega_{1}\right)$ is measured with a $10 \mathrm{GHz}$ two-tone RF signal spaced $10 \mathrm{kHz}$, when the output power of the RF signal is $-20 \mathrm{dBm}$. (a) The single MZM link. (b) The linearized link. 


\section{$59 \mathrm{dBc}$.}

The DWDPM linearized link is also constructed with the commercial components. The primary path is made up of an EM4 DFB laser operating at $1552.52 \mathrm{~nm}$ and a Covega MZM biased at + QUAD, while the secondary path comprises an Ortel DFB laser operating at $1535.82 \mathrm{~nm}$ and an MZM biased at + QUAD. The RF input signal is divided by a $6 \mathrm{~dB}$ RF power splitter and modulated on the two paths. An electric phase shifter is used to maintain opposite in phase between the inputs of the two MZMs. The optical signal outputs of the two paths are combined using a WM and injected into a DSC40S PD. With the proper adjustment of the input optical power of the secondary path, the IMD3 produced in this path can be equal in magnitude to those produced in the primary path. The IMD3 produced in the two paths are opposite in phase. Hence, the IMD3 of the linearized link can be suppressed with a penalty of a little reduction of the fundamental signal. The intermodulation $\left(2 \omega_{1}-\omega_{2}, 2 \omega_{2}-\omega_{1}\right)$ of linearized link is also measured, as shown in Fig. 3(b). The CIR of linearized link is $84 \mathrm{dBc}$ with the fundamental signal output power of $-20 \mathrm{dBm}$. Comparing with the intermodulation distortions $\left(2 \omega_{1}-\omega_{2}, 2 \omega_{2}-\omega_{1}\right)$ of the single MZM link, that of linearized link are suppressed $25 \mathrm{~dB}$.

The gain of the linearized link is $-22.4 \mathrm{~dB}$ and it is smaller by $9.8 \mathrm{~dB}$ than that of the single MZM link. This is attributed to the following reasons: (1) the RF power input of the linearized link is attenuated by $6 \mathrm{~dB}$ before inputting into the primary path because of using a $6 \mathrm{~dB}$ RF splitter; (2) the fundamental signal of the primary path subtracts that of the secondary path when suppressing the IMD3; (3) the RF gain of an optical link is proportional to the square of the output DC photocurrent, but due to the use of a wavelength multiplexer, the output DC photocurrent of the primary path in the linearized link is smaller than that for the single MZM link. The output DC photocurrent of the linearized link is $5.8 \mathrm{~mA}$ and that of the single MZM link equals $6.7 \mathrm{~mA}$. Furthermore, we measure the noise floors of both links with Agilent spectrum analyzer E4440A. The noise floor of the linearized link is equal to $-152.1 \mathrm{dBm} / \mathrm{Hz}$ and smaller than that of the single MZM link $(-150.8 \mathrm{dBm} / \mathrm{Hz})$, because the dominated noises of both links are proportional to the output DC photocurrent.

The SFDRs of both optical links are also measured. The measured data of both links are linearly fitted and the fitted lines are extrapolated, as shown in Fig. 4. As can be seen, the output fundamental signals of both links increase with the RF power input and their slopes are equal to 1 . Moreover, the intermodulation $\left(2 \omega_{1}-\omega_{2}, 2 \omega_{2}-\omega_{1}\right)$ of the single MZM link has a slope of 3 , while this slope of linearized link is 5 , which is caused by the residual IMD5 $\left(3 \omega_{1}-\omega_{1}-\omega_{2}, 3 \omega_{2}-\omega_{1}-\omega_{2}\right)$. Therefore, the IMD3 of the linearized link is suppressed and the residual IMD5 becomes the dominating distortion. According to the fitting lines, we calculate the input intercept point of the IMD3 (IIP3) of the single MZM link and the input intercept point of

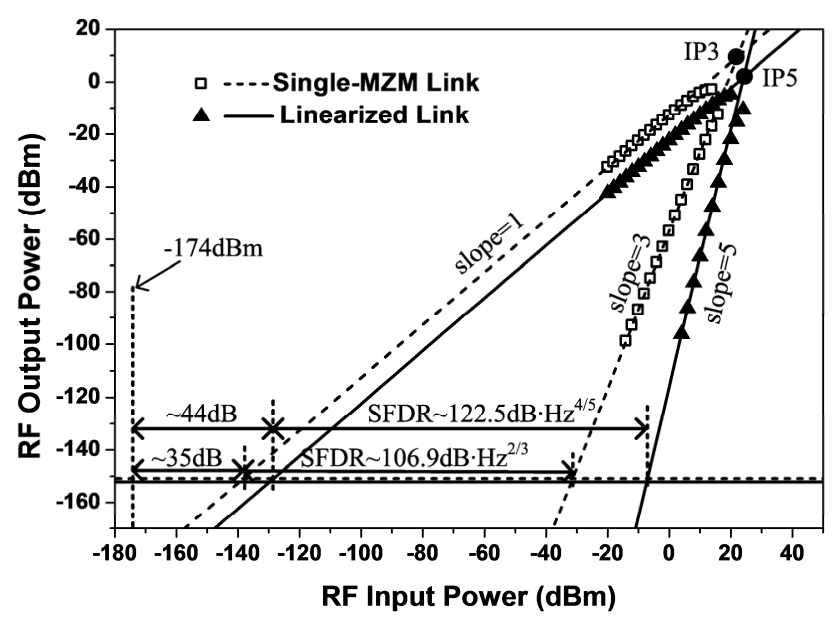

FIG. 4. Measured SFDRs of the single MZM link and the DWDPM linearized link.

the residual IMD5 (IIP5) of the linearized link, and they equal $22.1 \mathrm{dBm}$ and $23.5 \mathrm{dBm}$, respectively. The noise figures (NF) of both links are $35.7 \mathrm{~dB}$ and $44.3 \mathrm{~dB}$. In accordance with the computational formula of SFDR (Eq.3), the SFDRs of both links are calculated and their values are $106.9 \mathrm{dBHz}^{2 / 3}$ and $122.5 \mathrm{dBHz}^{4 / 5}$. Comparing with the SFDR of the unlinearized single MZM link, it is improved by $15.6 \mathrm{~dB}$ after linearization.

$$
\operatorname{SFDR}_{n}\left(d B \cdot H z^{n-1 / n}\right)=\frac{n-1}{n}\left(I I P_{n}-N F+174\right)
$$

According to the theory of Section 2, both the IMD3 and the residual HMD3 are proportional to $a_{3}$. Hence, if the IMD3 is suppressed, the residual HMD3 will also be suppressed and the linearity of the gain will be improved. We sweep and normalize the gains of the single MZM link and the linearized link with Agilent network analyzer. As shown in Fig. 5(a), when the RF power input is larger than $0 \mathrm{dBm}$, the gain of the single MZM link is compressed gradually, while that of the linearized link still keeps linear. Moreover, the RF power input required to achieve $1-\mathrm{dB}$ gain compression $\left(P_{R F, i n, l d B}\right)$ of the single MZM link is $12.7 \mathrm{dBm}$, and which of the linearized link is improved and equal $22.7 \mathrm{dBm}$.

The CDR is also an important performance metric of the optical links. It is the range of the input powers over which the signal is above the noise, and the gain is compressed by $1 \mathrm{~dB}$. The mathematical expression of the CDR is shown as below.

$$
C D R(d B \cdot H z)=\left(P_{R F, i n, 1 d B}-N F+174\right)
$$

According to the Eq.4, the CDRs of both links are measured, and equal $150.9 \mathrm{~dB} \cdot \mathrm{Hz}$ and $152.4 \mathrm{~dB} \cdot \mathrm{Hz}$, as shown in Fig. 5(b). Comparing with the $P_{R F, i n, l d B}$ and the 


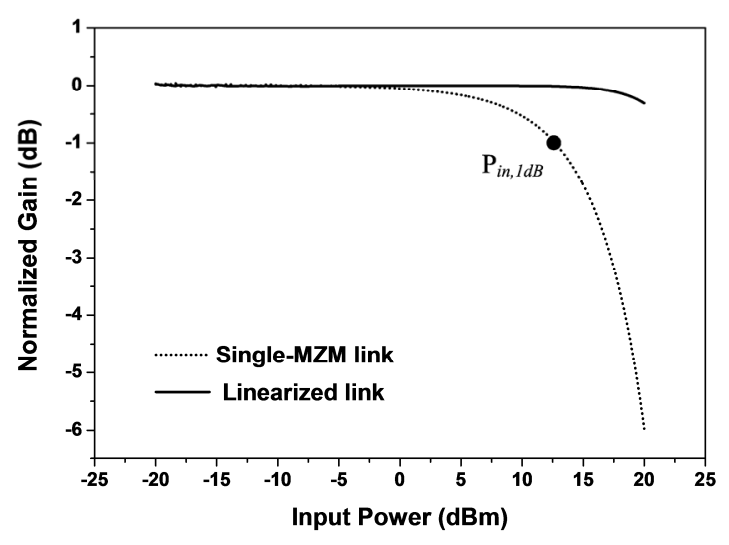

(a)

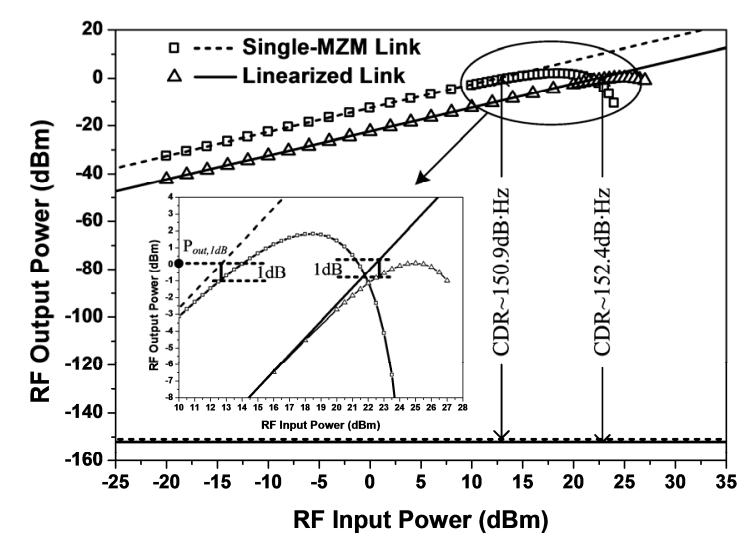

(b)

FIG. 5. (a) The normalized gains of the single MZM link and the DWDPM linearized link. (b) The CDRs of the single MZM link and the DWDPM linearized link.

CDR of the unlinearized single MZM link, which of the linearized link are improved by $10 \mathrm{~dB}$ and $1.5 \mathrm{~dB}$.

\section{CONCLUSIONS}

In this paper, we present and experimentally demonstrate the DWDPM linearization method. This DPM employing dual wavelengths prevents the use of an additional phase shifter and avoids the recreation of the intermodulation distortions due to the polarization coupling in the longhaul SMF. Moreover, the simulation results show that a high SFDR can be obtained readily by using this linearization method. We construct the DWDPM linearized link and the unlinearized single MZM link with the commercially available devices and compare the performance of both links. The experimental results show that the IMD3 of the linearized link is suppressed and the residual IMD5 becomes the dominating distortion. The intermodulation distortion of the linearized link is suppressed by $25 \mathrm{~dB}$ compared with that of the unlinearized single MZM link,. And the SFDR of the linearized link is now $122.5 \mathrm{~dB} \cdot \mathrm{Hz}^{4 / 5}$, which is improved by $15.6 \mathrm{~dB}$ after linearization. Furthermore, the $\mathrm{RF}$ power input required to achieve 1-dB gain compression and the CDR of the linearized link are also improved by $10 \mathrm{~dB}$ and $1.5 \mathrm{~dB}$, respectively.

\section{REFERENCES}

1. V. J. Urick, F. Bucholtz, J. D. McKinney, P. S. Devgan, A. L. Campillo, J. L. Dexter, and K. J. Williams, "Longhaul analog photonics," J. Lightwave Technol. 29, 11821205 (2011).

2. Y. Chiu, B. Jalali, S. Garner, and W. Steiner, "Broad-band electronic linearizer for externally modulated analog fiber-optic links," IEEE Photon. Technol. Lett. 11, 48-50 (1999).

3. A. Katz, W. Jemison, M. Kubak, and J. Dragone, "Improved radio over fiber performance using predistortion linearization," in Proc. 2003 IEEE MTT-S International Microwave Symposium Digest (Philadelphia, PA, USA, Jun. 2003), pp. 1403-1406.

4. V. J. Urick, M. S. Rogge, P. F. Knapp, L. Swingen, and F. Bucholtz, "Wide-band predistortion linearization for externally modulated long-haul analog fiber-optic links," IEEE Transactions on Microwave Theory and Techniques 54, 1458-1463 (2006).

5. J. A. MacDonald, M. V. Kuback, and A. Katz, "Wideband dynamic range improvement of microwave photonic links," in Proc. 2005 IEEE Conference-Avionics Fiber-Optics and Photonics (Minneapolis, MN, USA, Sep. 2005), pp. 67-68.

6. L. M. Johnson and H. Y. Roussell, "Reduction of intermodulation distortion in interferometric optical modulators," in Proc. Lasers and Electro-Optics Society Annual Meeting (Santa Clara, CA, USA, Nov. 1988), pp. 119-120.

7. L. M. Johnson and H. V. Roussell, "Linearization of an interferometric modulator at microwave frequencies by polarization mixing," IEEE Photon. Technol. Lett. 2, 810-811 (1990).

8. E. I. Ackerman, "Linearization of a broadband analog optical link using multiple wavelengths," in Proc. 1998 International Topical Meeting on Microwave Photonics (Princeton, NJ, USA, Oct. 1998), pp. 45-48.

9. E. I. Ackerman, "Broad-band linearization of a MachZehnder electrooptic modulator," IEEE Transactions on Microwave Theory and Techniques 47, 2271-2279 (1999).

10. G. E. Betts, "Linearized modulator for suboctave-bandpass optical analog links," IEEE Transactions on Microwave Theory and Techniques 42, 2642-2649 (1994).

11. G. E. Betts and F. J. O'Donnell, "Microwave analog optical links using suboctave linearized modulators," IEEE Photon. Technol. Lett. 8, 1273-1275 (1996).

12. S. K. Korotky and R. M. de Ridder, "Dual parallel modulation schemes for low-distortion analog optical transmission," IEEE J. Sel. Area Comm. 8, 1377-1381 (1990).

13. J. L. Brooks, G. S. Maurer, and R. A. Becker, "Implementation and evaluation of a dual parallel linearization system for AM-SCM video transmission," J. Lightwave Technol. 11, 34-41 (1993).

14. A. Karim and J. Devenport, "Low noise figure microwave photonic link," in Proc. IEEE/MTT-S International Microwave Symposium (Honolulu, HI, USA, Jun. 2007), pp. 1519-1522. 